\title{
Atom-Probe Tomography of Surface Oxides in a 20\% Cold Worked Stainless Steel Tested Under PWR Primary Water Conditions
}

\author{
S Lozano-Perez*, D W Saxey*, E A Marquis*, T Terachi** and T Yamada** \\ * Department of Materials, University of Oxford, Parks Road, Oxford, OX1 3PH, UK \\ ** INSS, 64 Sata, Mihama-cho, Mikata-gun, Fukui 919-1205, Japan
}

Low potential stress corrosion cracking (LPSCC) of stainless steels is a very important degradation phenomenon in pressurized water reactors (PWRs). Although SCC of austenitic steels (304 and 316) has not been reported yet in operating PWR plants (except from irradiated materials), careful autoclave testing in under controlled environments have confirmed that highly cold-worked stainless steels are susceptible to SCC even in PWR primary water conditions [1]. In order to clarify the corrosion mechanisms and the role of cold work, it is important to characterize the oxide-matrix interface with the highest level of detail. It is well known that the oxide films formed in steels under these conditions consist of a double-layer structure, with an inner Cr-rich spinel and an outer Fe-rich spinel layer. In Figure 1, a STEM HAADF image is shown together with an EELS line profile across the oxide layers and into the matrix. Data was acquired in a Jeol $3000 \mathrm{~F}$ operated at $297 \mathrm{kV}$ equipped with a GIF. Probe size was $\sim 1 \mathrm{~nm}$ and sampling distance $5 \mathrm{~nm}$. Collection and convergence angles were 26 and $16 \mathrm{mrad}$ respectively. Gatan DM's was used for quantification.

Although high-resolution analytical (S)TEM is a powerful technique, it is crucial to understand how the oxidation progresses in 3D and interacts with the deformed microstructure created by the cold work, which consist mostly of families of deformation with a complex internal cellular structure and high local dislocation densities. For this reason, specimens were prepared for atom-probe tomography (APT) containing original surface oxide layers. Details on alloy composition and testing details can be found in [2]. A FEI 200 single beam FIB was used for the preliminary sample preparation, while a Zeiss NVision 40 was used for the final milling and sharpening [3]. Samples were prepared as $20 \mu \mathrm{m}$ long posts which were welded to Fischione posts (for holder model 2050) for dual TEM/APT examination and finished with a $2 \mathrm{kV} \mathrm{Ga}^{+}$final cleaning (See Fig 2).

The surface-oxide specimen was analysed in a LEAP $3000 \mathrm{HR}^{\mathrm{TM}}$ atom probe using $\sim 10 \mathrm{ps}$ laser pulses to stimulate field evaporation. The laser spot was approximately $5 \mu \mathrm{m}$ in diameter, with a wavelength of $532 \mathrm{~nm}$, a pulse rate of $160 \mathrm{kHz}$, and approximately $0.7 \mathrm{~nJ}$ per pulse. The specimen base-temperature was $65 \mathrm{~K}$ during the analysis. Reconstructed volume clearly shows that the inner Cr-rich spinel was preserved during evaporation and that the deformation introduced by the cold work is being preferentially oxidized (Figure 3). Oxide and matrix compositions are consistent with the EELS data (Fig 1), demonstrating the validity of the approach and lack of major artifacts from the evaporation. A small $\mathrm{Cu}$ segregation to the interface which was not previously detected is also reported.

[1] K. Arioka et al. Corrosion 62[1], p.74, (2006).

[2] S. Lozano-Perez et al. J Nuc Mat 374, 61-68 (2008).

[3] D. W. Saxey et al. Ultramicroscopy 107, 756-760 (2007).

[4] S. Lozano-Perez is grateful to INSS for sponsoring this research. 

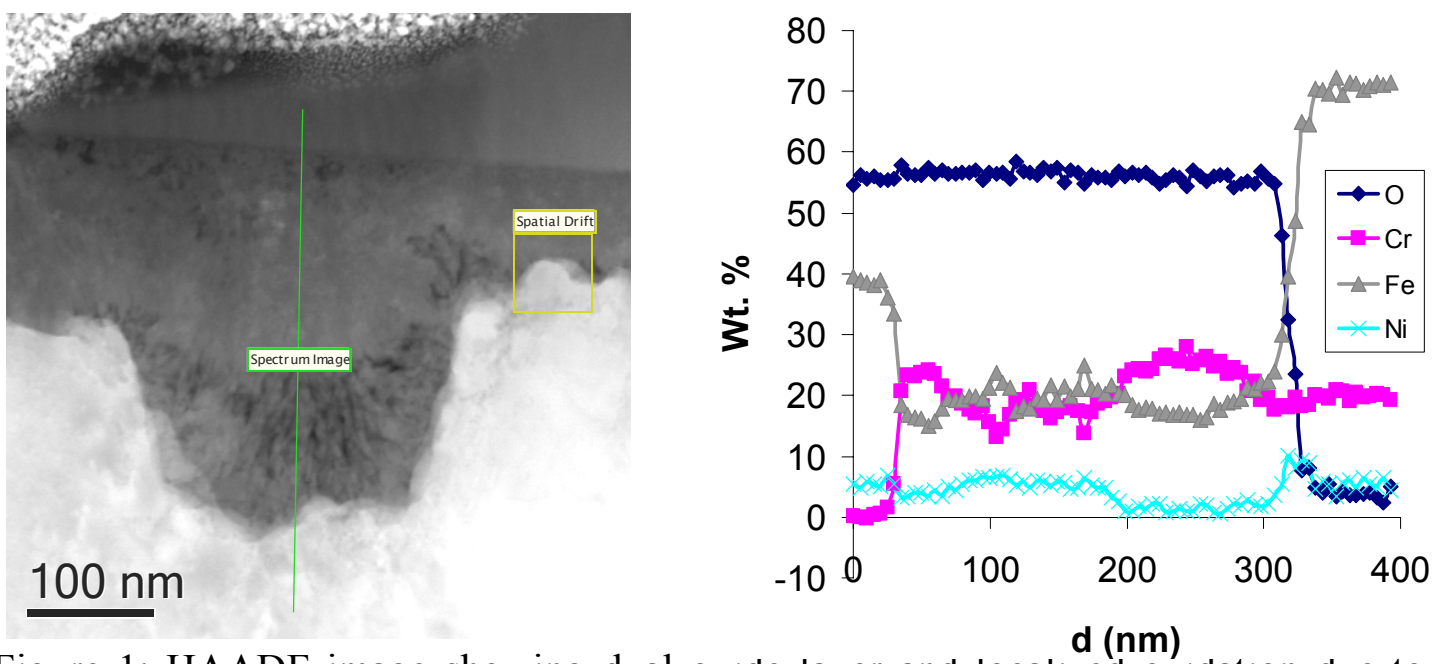

Figure 1: HAADF image showing dual oxide layer and localized oxidation due to cold work (Pt layer from FIB is visible on the top) (left); Quantitative EELS line profile showing changes in composition across the layers and into the matrix (right).
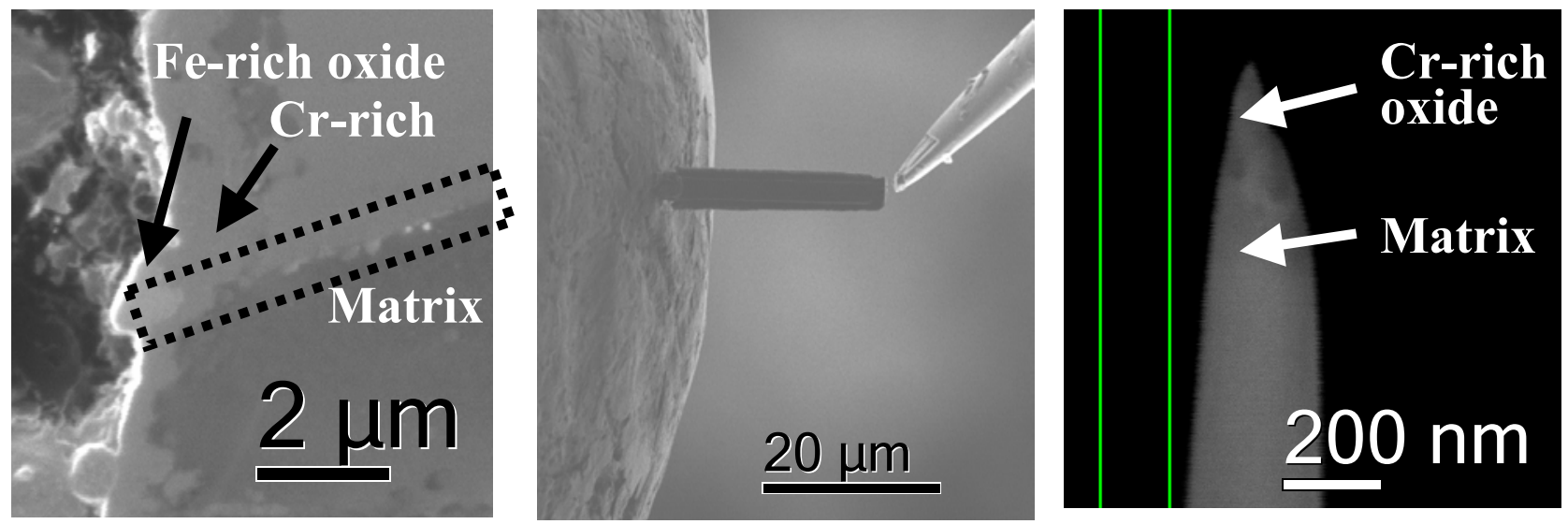

Figure 2: FIB SE image showing selection of volume containing oxide (left); FIB SE image showing welding to Fischione post (centre); SEM In-lens image showing the final shape of an APT sample.
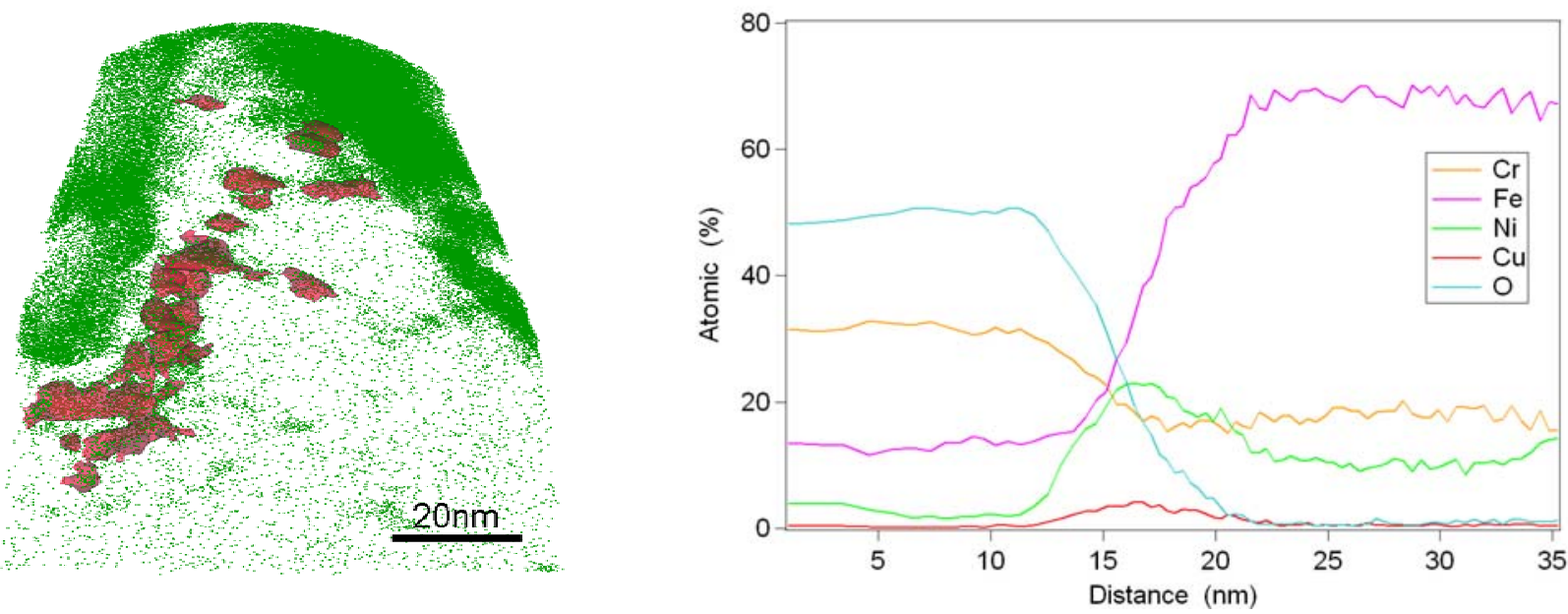

Figure 3: APT reconstructed volume showing Cr-oxides (green) in direct contact with the surface, and isoconcentration surfaces (red) of oxidized deformation bands (also Cr-rich oxide) (left); Quantitative 1-D profiles across Cr-rich oxide / matrix interface (right). 